\title{
Hot-cross bun sign as a neuroradiological clue to multiple system atrophy with predominant cerebellar ataxia
}

Joseph Bruno Bidin Brooks ${ }^{1 *}$, Fábio César Prosdócimi², Rodrigo André Oliveira ${ }^{3}$, Guilherme Lopes da Silveira ${ }^{3}$, Fernando Pierini Costa Willian Thiago Almeida Leite ${ }^{5}$ and Camila Salles Lopes ${ }^{6}$

${ }^{1}$ Irmandade Santa Casa Misericórdia de Santos- Department of Neurology; UNIMES- Universidade Metropolitana de Santos, Department of Structure and Function ${ }^{2}$ UNIMES- Universidade Metropolitana de Santos, Department of Structure and Function

${ }^{3}$ Clínica Mega Imagem

${ }^{4}$ Irmandade Santa Casa Misericórdia de Santos- Department of Neurology

${ }^{5}$ Irmandade Santa Casa Misericórdia de Santos- Department of Neurology

${ }^{6}$ Medical student; UNIMES- Universidade Metropolitana de Santos

\begin{abstract}
Multiple system atrophy with predominant cerebellar ataxia (MSA-c) is rare neurodegenerative characterized by asymmetrical cerebellar ataxia, autonomic dysfunction, predominating in urogenital, respiratory and cardiovascular domains. The present case refers to a 36-year-old woman with progressive gait instability, postural hypotension and urinary incontinency. Brain MRI showed cerebellar and pons atrophy and the "hot cross bun" sign providing a clue to its possible etiology. Laboratorial and cerebrospinal fluid examination were normal. Symptomatic treatment was instituted with partial improvement of the symptoms. Attention to this clinical-radiologic correlation may help physicians make correct diagnoses and appropriate treatment.
\end{abstract}

\section{Introduction}

Multiple system atrophy with predominant cerebellar ataxia (MSA-c) is rare sporadic neurodegenerative disease that affects equally both gender and middle aged patients. This disease is characterized by motor and nonmotor symptoms; The motor findings include asymmetrical cerebellar ataxia, pyramidal dysfunction, focal dystonia, dysphonia, dysarthria and dysphagia. The nonmotor findings include autonomic dysfunction, predominating in urogenital, respiratory and cardiovascular domains. Frontal-lobe attention deficiency, emotional incontinence and behavioral changes can occur and may be also related to chronic pain $[1,2]$. Here, we present a case of MSA-c with Magnetic Resonance Imaging (MRI) findings.

\section{Methods}

The report of this case was submitted and approved by the ethics committee of Universidade Metropolitana de Santos.

\section{Case presentation}

The present case refers to a 36-year-old caucasian woman with a 2-year history of progressive gait instability and slurred speech. She also had postural hypotension, sleep behavior disorder and urinary incontinency. Neurological examination revealed axial ataxia, nystagmus and orthostatic hypotension. There was no head trauma and her medical history was unremarkable. Sagittal $\mathrm{T}^{\star}$ and Axial FLAIR weighted brain MRI showed cerebellar and pons atrophy and the "hot cross bun" sign (white arrows) providing a clue to its possible etiology (Figure 1). Laboratorial and cerebrospinal fluid examination were normal. Symptomatic treatment was instituted with partial improvement of the symptoms.
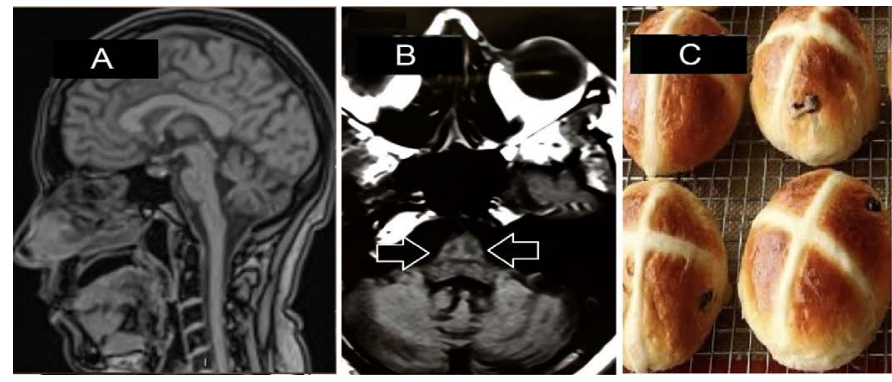

Figure 1. A- Sagittal T1* weighted brain MRI showing cerebellar and pontine atrophy. B- Axial FLAIR* exhibit the "hot cross bun" sign in the pons and cerebellar atrophy (white arrows). C- Hot cross bun served during Holy Week.

\section{Discussion}

The most frequent imaging findings in MSA-c are cerebellar and pons atrophy, with compensatory enlargement of the IV ventricle and cerebellum-pontine cisterns. The cruciform hypersignal in T2ECHO and FLAIR weighted MRI, it may be associated to wallerian degeneration and gliosis of cerebellar transverse fibers (horizontal axis) and raphe neurons of the pons (vertical axis), preserving the tegmentum and the cortico-spinal tracts. This radiological signal it is also known as "hot cross bun" and it's related due to the similarity to

${ }^{*}$ Correspondence to: Joseph Bruno Bidin Brooks, Irmandade Santa Casa de Misericórdia de Santos, Avenida Cláudio Luiz da Costa 50, Santos, 11075-900, São Paulo, Brazil, Tel: +55-13-32020600; E-mail: joseph3b@gmail.com

Key words: multiple system atrophy, brain imaging, case report

Received: July 03, 2018; Accepted: July 12, 2018; Published: July 16, 2018 
a popular bread served during Holy Week in United Kingdom. The radiological differential diagnosis are spinocerebellar ataxias (SCA), the X-fragile mutation (FXTAS) and inflammatory vasculitis affecting the brainstem.

\section{Conclusion}

This case report alerts to the possibility of MSA-c in the differential diagnosis of patients with progressive cerebellar ataxia, pyramidal and autonomic dysfunction. Attention to this clinical-radiologic correlation may help physicians make correct diagnoses and appropriate treatment.

\section{References}

1. Das B, Patil A, Goyal MK (2016) Hot cross bun sign. QJM 109: 203-204.

2. Fanciulli A, Wenning GK (2015) Multiple-system atrophy. N Engl J Med 372: 249-263.

Copyright: $\odot 2018$ Roever L. This is an open-access article distributed under the terms of the Creative Commons Attribution License, which permits unrestricted use, distribution, and reproduction in any medium, provided the original author and source are credited. 